\title{
The "AIDS Presenters" phenomenon, thirteen years after the availability of potent, combination antiretroviral therapy
}

Roberto Manfredi

From $16^{\text {th }}$ International Symposium on HIV and Emerging Infectious Diseases

Marseille, France. 24-26 March 2010

\section{Background}

Notwithstanding the availability of potent, combined antiretroviral therapy (cART), AIDS notifications continue to occur, with increasing prevalence for patients (p) who missed or neglected their condition, or refused or took with insufficient compliance the recommended antiretroviral medications.

\section{Methods}

All cases of AIDS notified since the year 2001 were compared with those found in the decade preceding cART availability (1986-1995).

\section{Results}

Compared with the pre-cART era, a significant drop of frequency of overall AIDS cases occurred: from a mean $58.3 \pm 11.2$ patients-year observed in the decade 19861995 , to $13.7 \pm 6.0$ patients-year during years 2001-2008 $(p<.001)$, together with an increased mean age $(p<$ $.002)$, female gender $(p<.01)$, sexual vs i.v. transmission $(p<.001)$, and proportion of immigrant versus native $\mathrm{p}$ $(p<.02)$. In the cART era, the most evident drop of frequency interested opportunistic diseases linked to a CD4 + lymphocyte count below $50-100$ cells/ $\mu \mathrm{L}$, while a proportional rise of tuberculosis, pneumonia, lymphomas, and other neoplasms was observed. The frequency of both Candida esophagitis and Pneumocystis carinii pneumonia remained stable, as the first two most frequent AIDS-related conditions. After cART availability, the following diagnoses in crude frequency were represented by neurotoxoplasmosis, wasting syndrome, AIDS-dementia complex, and non-Hodgkin's lymphomas. P with multiple
AIDS-defining diseases, and also AIDS diagnoses made only at or after death, even showed a paradoxically increased frequency and absolute number during the cART era versus the prior decade $(p<.001$ and $p<.03)$, while no difference was found as to the grade of HIVassociated immunodeficiency. Surprisingly, an underlying anti-HIV therapy was a more common event until 1995, versus $\mathrm{p}$ observed in the cART era $(p<.001)$, since during recent years AIDS notification tends to be increasingly associated with the first diagnosis of HIV infection.

\section{Discussion}

When facing $\mathrm{p}$ with some form of opportunism, clinicians should maintain an elevated suspect for an advanced (but missed-untreated) HIV disease. A continued level of attention will help a more rapid recognition and an appropriate management of $\mathrm{p}$ who could not take benefit from cART, since they remained unaware of their disease, or refused controls and treatment during the previous years.

Published: 11 May 2010

doi:10.1186/1742-4690-7-S1-P34

Cite this article as: Manfredi: The "AIDS Presenters" phenomenon,

thirteen years after the availability of potent, combination antiretroviral therapy. Retrovirology 2010 7(Suppl 1):P34.

Correspondence: Roberto.manfredi@unibo.it

Infectious Diseases, University of Bologna, S. Orsola Hospital, Bologna, Italy 